\title{
Aspen/Snowmass Perinatal Biology Symposium: "Interconnecting Animal and Human Systems to Understand Life-Long Disease"
}

\author{
August 27-30th 2016, Snowmass Colorado \\ Organizing Committee: \\ Paul Rozance, MD, University of Colorado School of Medicine \\ Tim Regnault, $\mathrm{PhD}$, Western University \\ Kim Vonnahme, PhD, North Dakota State University
}

The Aspen/Snowmass Perinatal Biology Conference is a multidisciplinary research meeting that brings together clinicians and scientists, established senior and junior beginning investigators and graduate students to report and discuss their findings in an atmosphere conducive to frank yet amicable exchange. The overall goal is to develop effective trans-disciplinary scientific interactions that lead to productive translational perinatal science research endeavors.

\section{Sponsorship Statement}

Publication of this supplement was sponsored by the Division of Neonatology, University of Colorado School of Medicine.

doi:10.1038/pr.2016.141

\section{ORAL SESSION}

O1: Impaired hind limb glucose oxidative capacity in intrauterine growth restricted lambs LE Camacho ${ }^{1}$, DT Yates ${ }^{2}$, MA Davis ${ }^{1}$, AC Kelly $^{1}$, and SW Limesand ${ }^{1}$

${ }^{1}$ The University of Arizona, Tucson, AZ, USA. ${ }^{2}$ University of Nebraska-Lincoln, Lincoln, NE, USA Evidence shows that intrauterine growth restriction (IUGR) increases the risk for impaired glucose tolerance in adulthood. The objective of this study was to determine whole-body glucose disposal rates and hind limb glucose metabolism in lambs with placenta insufficiency-induced IUGR. At 28 days, glucose disposal rates and insulin sensitivity were measured with [14C(U)]-D-glucose at basal and hyperinsulinemic-euglycemic steady state periods. Moderate and high steady state insulin concentrations were induced by infusing insulin (HumalinR) at 2 and $4 \mathrm{mU} / \mathrm{min} / \mathrm{kg}$. IUGR lambs were lighter $(\mathrm{P}=0.02)$ at birth than controls $(3.3 \pm 0.5 \mathrm{vs} 4.3 \pm 0.3 \mathrm{~kg})$. Although basal insulin concentrations were similar between treatments, IUGR lambs had higher $(\mathrm{P}<0.01)$ insulin concentrations compared to controls during the moderated $(10.3 \mathrm{vs} .5 .6 \pm 1.7 \mathrm{ng} / \mathrm{mL})$ and high $(21.1 \mathrm{vs}$ $13.4 \pm 1.7 \mathrm{ng} / \mathrm{mL}$ ) periods. However, glucose concentrations were not different between treatments or periods. Whole-body glucose disposal rate was similar between treatments but increased during the hyperinsulinemic periods. Hind limb glucose uptake was greater $(\mathrm{P}<0.05)$ in IUGR lambs compared to controls, and the rates increased with hyperinsulinemia. Rates of hind limb glucose oxidation were not different, but the fraction of glucose oxidation to utilization was reduced $(\mathrm{P}<0.01)$ in IUGR lambs $(10.5 \pm 1.4 \%)$ compared to controls $(16.2 \pm 1.0 \%)$. Two major outcomes from this study were that insulin clearance was impaired in IUGR lambs and that even though whole body glucose disposal is not altered at this age hind limb fractional glucose oxidation is impaired. The deficiency in fractional glucose oxidation indicates that mitochondria oxidative capacity in peripheral insulin responsive tissues is disrupted.

Support: Bill and Melinda Gates Foundation, Global Health Grant \# OPP1066912.

O2: Chronic ischemia augments coronary reserve in fetal myocardium

E Chang ${ }^{1}$, S Louey ${ }^{1}$, I Lindgren ${ }^{1}$, G Giraud ${ }^{1}$, and K Thornburg ${ }^{1}$

${ }^{1}$ Oregon Health and Science University, Portland, Oregon, USA

Objective: The mammalian fetus is susceptible to moderate-severe ischemic events which may hamper normal myocardial development. At present, there are no animal models to study blood flow reduction in immature myocardium. We have developed a chronic coronary occlusion model in fetal sheep. We tested the hypothesis that chronic reduction in coronary flow stimulates coronary microvascular growth and augments flow reserve.

Methods: Four ovine fetuses (123 \pm 2 dGA, term 147 d) were instrumented with vascular catheters and a circumflex coronary Doppler flow probe was placed upstream from a vascular occluder. Circumflex flow was progressively reduced to $50 \%$ over five days. Coronary conductance curves were constructed before and after the five days of partial occlusion, with and without adenosine infusion but while the occluder was deflated. We analyzed data using analysis of covariance.

Results: Fetal myocardial flow autoregulated between driving pressures of $30-50 \mathrm{mmHg}$. Prior to partial coronary occlusion, adenosine increased mean flow reserve by $475 \mathrm{ml} / \mathrm{min} / 100 \mathrm{~g}$ above resting flow at $40 \mathrm{mmHg}(\mathrm{P}<0.001)$. After five days of reduced coronary flow, resting conductance curves remained unchanged compared to pre-occlusion. However during adenosine infusion, mean flow reserve at $40 \mathrm{mmHg}$ approximately doubled $(930 \mathrm{ml} / \mathrm{min} / 100 \mathrm{~g} ; \mathrm{P}<0.001)$.
Conclusion: Chronic fetal myocardial ischemia leads to a near doubling of coronary flow reserve, in contrast to myocardial response to anemia, where conductance is dramatically augmented both with and without adenosine. In both models, elevated maximal conductance indicates a powerful remodeling of coronary microvascular elements. These data suggest that augmented sheer forces in the coronary vessels, as seen in anemia but not in the ischemic model, are not required for vascular remodeling in the near-term myocardium.

Support: NIH 5P01HD034430 and 5T32HL094294.

O3: Glutamate transfer (clearance) by the placenta adapts according to placental size in mice in late gestation

K McIntyre $^{1}$, C Hayward ${ }^{1}$, C Sibley ${ }^{1}$, S Greenwood ${ }^{1}$, and M Dilworth

${ }^{1}$ University of Manchester, UK

Objective: Appropriate fetal growth is dependent upon adequate placental nutrient transfer yet we still do not fully understand the mechanisms how these are matched. Studies in mice have demonstrated that the lightest placentas within a wild-type litter adaptively up-regulate transfer (in terms of, for example, volume of plasma cleared per gram of placenta) via the system A amino acid transporter, putatively to compensate for their reduced size and maintain fetal growth on a normal trajectory. Metabolism of glutamate in the placenta is a key source of glutamine for fetal growth. Here, we tested the hypothesis that there is adaptive up-regulation of the placental transfer of glutamate in relation to size of placenta.

Methods: Pregnant C57Bl6/J (wild-type) dams were injected with radiolabelled (14C) glutamate via the tail vein at embryonic day $(\mathrm{E}) 15.5(\mathrm{n}=6)$ and $\mathrm{E} 18.5(\mathrm{n}=10$ term=E19.5). At approximately 2 minutes post-injection, dams were sacrificed, fetal and placental tissues weighed and unidirectional maternofetal clearance (14C-glutamateKmf) calculated ( $\mu$ l per g placental tissue). Expression of the glutamate transporter protein EAAT2 (system XAG-) was determined by Western blot analysis.

Results: The lightest placentas were on average $24 \%$ lighter than the heaviest placentas within a litter. At E15.5 fetuses with lightest placentas weighed $87 \%(\mathrm{P}<0.05)$ of fetuses with the heaviest placentas but were similar at E18.5. 14C glutamateKmf was significantly greater in the lightest placentas at $\mathrm{E} 18.5$ only $(133 \% \mathrm{P}<0.05)$. Comparing lightest and heaviest placentas, there was no difference in expression of EAAT2 transporter protein (per mg membrane protein).

Conclusion: Adaptive regulation of placental glutamate transfer in the lightest placentas compared to heaviest placentas in a litter was observed in late gestation. An increase in glutamate transporter activity, rather than expression of EAAT2, may underlie this adaptation. Future work will explore mechanisms of adaptation and address the hypothesis that failure of adaptation may contribute to complications such as fetal growth restriction.

Support: MRC Fellowship Grant MR/K024442/1.

O4: Early gestation intrauterine hypoxia causes placental insufficiency and maternal hypertension in pregnant guinea pig model

L Pence ${ }^{1}$, LP Thompson ${ }^{2}$, G Pinkas ${ }^{2}$, BP Telugu ${ }^{1}$

${ }^{1}$ University of Maryland, College Park, MD, USA; ${ }^{2}$ University of Maryland, Baltimore, USA

Objective: Pregnant guinea pig (G.Pig) is an ideal model for investigating the mechanisms mediating human placental development. Similar to humans, G.Pig has a hemochorial placenta, a villous 
subplacenta analogous to cell columns of human placenta, and a characteristic deep invasion and remodeling of maternal spiral arteries (SA). Using the pregnant G.Pig model, the objective of this study was to test the hypothesis that chronic intrauterine hypoxia stimulates proliferation but inhibits trophoblast (TB) invasion and SA remodeling.

Methods: Time-mated pregnant G.Pigs were exposed to either normoxic (NMX) or hypoxic (HPX; $10.5 \% \mathrm{O}_{2}$ ) conditions at $25 \mathrm{~d}$ gestation (implantation: $23 \mathrm{~d}$ ) until mid-term (39-40d) or near-term (60-65d). From euthanized animals placentas were excised, weighed, and RNA extracted for qRTPCR analysis, or perfusion-fixed with formalin for immunohistochemistry for TB (KRT7), blood vessels (SMA-smooth muscle actin, vWF-endothelium), and proliferation (PCNA) in the placenta and associated uterine tissue.

Results: At term, hypoxia increased mean maternal arterial blood pressure (HPX $57.9 \pm 2.3 \mathrm{vs}$ NMX $40.4 \pm 2.3, \mathrm{p}<0.001)$, decreased fetal weight by $16.1 \%(\mathrm{p}<0.05)$, and increased absolute and relative placenta to fetal weight by $10.1 \%$ and $31.8 \%$, respectively $(\mathrm{p}<0.05)$. At mid-term, there was a significant increase in TB in HPX placentas as confirmed by elevated ESX1 gene expression $(\mathfrak{p}<0.05)$, and staining for PCNA and KRT7. Image analysis of the decidua revealed that HPX placentas had a $39.2 \%$ decrease in the KRT7:vWF signal ratio, and evaluation of individual maternal vessels showed reduced occupation and remodeling by KRT7 positive TB in HPX placentas.

Conclusion: The HPX placenta compensates maladaptively by stimulating TB proliferation but inhibiting invasion into the SA. We conclude that oxygenation of the placenta regulates norma SA remodeling and that HPX conditions contribute to inadequate blood flow and placental insufficiency leading to maternal hypertension and fetal growth restriction. This is the first evidence of hypoxia-induced repression of SA remodeling in guinea pig placenta.

Support: UMB-UMCP Seed Grant to LT and BT, and NIH HL126859 to LT.

O5: Cardiometabolic risk in the offspring of leptin receptor mutation heterozygous pregnancy J Thompson ${ }^{1}$, J Mintz ${ }^{1}$, B Stansfield

'Augusta University, Augusta, Georgia, USA

Objective: To develop a new model to study the impact of gestational diabetes (GDM) on metabolic function in the offspring.

Methods: Wild type (Wt) and female mice heterozygous for the leptin receptor mutation (Het were mated with Wt males. Metabolic parameters in Wt and Het ${ }_{d b}$ females were evaluated before conception, at Gd17 (term = Gd19) of pregnancy and 4-weeks after pregnancy. For offspring studies, only Wt offspring were assessed. Glucose tolerance was evaluated in fasted mice after IP injection of glucose, fasting plasma lipid and insulin levels measured using Colorimetric assays and body composition determined by NMR spectroscopy.

Results: Prior to conception, non-pregnant Het females displayed increased adiposity and reductions in lean body mass relative to age-matched controls $(\mathrm{p}<0.001)$, yet had normal regulation of blood glucose. On Gd17 there was an increase in maternal fasting blood glucose, insulin and triglyceride levels, and a reduction in glucose clearance in pregnant Het $v$ vs. Wt females (all p < 0.05). Pregnancy-induced glucose intolerance in $\mathrm{Het}_{d b}$ females resolved 4-weeks post-pregnancy. At 3 -weeks of age, \% body fat was increased in male $(\mathrm{p}<0.001)$ and female $(\mathrm{p}<0.001)$ Wt offspring born from a Het dam, and remained higher at 3-months and 6-months of age $(\mathrm{p}<0.05)$. At 6-months, fasting plasma triglyceride $(\mathrm{p}<0.01)$, cholesterol $(\mathrm{p}<0.05)$ and non-esterified fatty acids $(\mathrm{p}<0.01)$ were increased in Wt offspring of $\mathrm{Het}_{d b} \mathrm{vs}$. Wt pregnancies.

Conclusion: Similar to human GDM, the Het female has high pre-pregnancy adiposity and spontaneously develops glucose intolerance, hyperinsulinemia and hypertriglyceridemia during pregnancy. Further, Het ${ }_{d b}$ pregnancy appears to program an abnormal metabolic phenotype in the offspring, independent of genotype. Therefore, the Het ${ }_{d b}$ female is a valuable model for the study of GDM that has remains virtually unexploited for the study of fetal programing.

Support: American Heart Association.

O6: Hind limb amino acid uptake and hind limb growth rates are reduced in the late gestation IUGR fetus

L Zastoupil $^{1}$, AC Blake ${ }^{1}$, PJ Rozance ${ }^{1}$, SR Wesolowski ${ }^{1}$, WW Hay Jr ${ }^{1}$, RB Wilkening ${ }^{1}$, LD Brown ${ }^{1}$ ${ }^{1}$ University of Colorado, Aurora, CO, USA

Objective: Intrauterine growth restriction (IUGR) fetuses from placental insufficiency develop reduced muscle mass that does not fully recover by adulthood. Our objective was to determine the effect of chronic placental insufficiency on fetal skeletal muscle cell proliferation, growth, and substrate metabolism during late gestation.

Methods: Surgery was performed to measure hind limb length, followed by catheterization of the hind limb with aortic (a) and femoral venous (v) sampling catheters in IUGR ( $\mathrm{n}=11-12)$ and control $(n=15-18)$ fetal sheep. An ultrasonic flow transducer was placed around the external iliac artery. One week after surgery, the following measurements were determined: hind limb blood flow (normalized to $100 \mathrm{~g}$ hind limb weight), substrate uptake rates [(a-v) $\mathrm{x}$ hind limb flow], muscle cell proliferation using fetal BrdU infusion, and hind limb muscle weights and growth rates.

Results: In IUGR fetuses, hind limb muscle weights normalized to hind limb length were $\sim 35 \%$ lower $(P<0.005)$ and hind limb growth rates were $46 \%$ lower than control (IUGR $0.26 \pm 0.07$, control $0.48 \pm 0.07 \mathrm{~cm} /$ day; $P<0.05$ ). Hind limb flow was maintained in IUGR (IUGR $18.1 \pm 1.8$, control $15.3 \pm 1.1 \mathrm{ml} / \mathrm{min} / 100 \mathrm{~g}$ ). Hind limb glucose uptake rates were maintained in IUGR (IUGR 2.1 \pm 0.3 , control $2.4 \pm 0.1 \mu \mathrm{mol} / \mathrm{min} / 100 \mathrm{~g}$ ) and glucose extraction efficiency was higher (IUGR 20.2 \pm 1.2 control $17.2 \pm 0.8 \% ; P<0.05$ ). Oxygen uptake rates tended to be lower in IUGR (IUGR $11.3 \pm 1.3$ CON $14.1 \pm 1.0 \mu \mathrm{mol} / \mathrm{min} / 100 \mathrm{~g} ; P=0.09$ ) and hind limb lactate output tended to be higher (IUGR $1.5 \pm 0.2$, control $0.9 \pm 0.2 \mu \mathrm{mol} / \mathrm{min} / 100 \mathrm{~g} ; P=0.09$ ). Total amino acid uptake by the hind limb was lower in IUGR (IUGR $1.3 \pm 0.5$, control $2.5 \pm 0.3 \mu \mathrm{mol} / \mathrm{min} / 100 \mathrm{~g} ; P<0.05$ ) and cellular proliferation within the biceps femoris muscle also was decreased (IUGR $1.1 \pm 0.1$, control $2.8 \pm 0.7 \% \mathrm{BrdU}+$ total nuclei; $P<0.05$ )

Conclusion: Skeletal muscle adapts to chronic placental insufficiency in the IUGR fetus by decreasing amino acid uptake, which likely contributes to reduced hind limb growth, muscle mass accretion, and cellular proliferation. We speculate that oxidative metabolism within the fetal hind limb decreases concurrently with a reduction in muscle growth, but glucose uptake rates are maintained to support basal energy requirements.

\section{POSTER SESSION 1 - MATERNAL AND PLACENTAL REGULATION}

P1-1: Maternal nutritional factors associated with inflammation in preeclampsia

CM Anderson ${ }^{1}, \mathrm{~A} \mathrm{Tan}^{1}$, D Perley ${ }^{2}$, JE Ohm²

${ }^{1}$ The Ohio State University, Columbus, OH, USA, ${ }^{2}$ University of North Dakota, Grand Forks, ND, USA Objective: Preeclampsia (PE) is a pregnancy-specific condition associated with inflammatory changes that present prior to symptom development. Inflammation etiology is unknown though has been linked to elevated C-reactive protein (CRP), a nonspecific marker of the innate immune system. Variants in the CRP gene increase the risk for preeclampsia though there is little known regarding epigenetic changes and related maternal factors that influence CRP levels in preeclampsia. The purpose of this study was to examine the association between circulating CRP, dietary inflammatory potential, maternal weight and CRP DNA methylation in preeclampsia. Our central hypothesis is that elevated CRP levels are associated with CRP gene hypomethylation, increased maternal weight and inflammatory dietary intake in preeclampsia.

Methods: Prospectively, we collected blood from nulliparous women $(n=40)$ in each of three trimesters of pregnancy for measurement of serum CRP and DNA methylation (450K Illumina Infinium). Dietary intake in each of three trimesters was collected using a food frequency questionnaire, with inflammatory potential of diet determined by using the Dietary Inflammation Index (DII). Maternal gestational weight gain and pregnancy outcome (normotensive pregnancy [NP], $n=34$; preeclampsia $[\mathrm{PE}], \mathrm{n}=6$ ) were determined through medical record abstraction. Analysis included descriptive statistics, $t$-test for group differences, and logistic regression for effects of CRP, DII and gestational weight gain on the risk for PE. Significance was determined at $\mathrm{p}<0.05$.

Results: CRP was increased across pregnancy in women with PE, with significant group differences limited to second trimester $(\mathrm{p}<0.05)$. While DII did not differ among NP and PE women, a reduction in omega 3 dietary intake was identified in women with $\mathrm{PE}$ in the second trimester. There were no differences in CRP DNA methylation though methylation gain (HSPG2, IGSF21, SEMA4D) and loss (HLA-A, MYOM2, MYBPH) in genes regulating immune function were identified in women with PE. While there was no significant effect of DII score or CRP in PE, gestational weight gain increased risk for PE (OR 1.13, $\mathrm{p}<0.02)$.

Conclusion: Mid pregnancy CRP increase, decreased omega3 dietary intake and increased gestational weight gain were associated with pregnancy complicated by PE. Differential DNA methylation in immunologic pathways implicate maternal nutritional factors as targets for future investigation.

Support: NIH 1P20GM104360-01, RWJF 64202.

P1-2: Immunofluorescence localization of estrogen receptors in sheep utero-placenta during early pregnancy: Effects of assisted reproductive technologies (ART)

S. Bairagi ${ }^{1}$, AT Grazul-Bilska ${ }^{1}$, PP Borowicz ${ }^{1}$, LP Reynolds ${ }^{1}$

${ }^{1}$ North Dakota State University, Fargo, ND, USA

Objective: Steroids mediate utero-placental vascular development and remodeling, which is critical for successful establishment of pregnancy. We hypothesized that expression of estrogen receptors (ESR) 1 (alpha) and 2 (beta) would change during early pregnancy and after application of ART.

Methods: In Experiment 1, utero-placental tissues were collected from days 14 to 30 after mating (pregnant) and on day 10 after estrus (non-pregnant controls) $(n=4 /$ day). In Experiment 2 utero-placental tissues were collected on Day 22 after natural mating (NAT) or after application of ART including embryo transfer (NAT-ET), in vitro fertilization (IVF) or in vitro activation (IVA) $(n=3-5 /$ group). Localization of ESR in uteroplacental tissues was determined using immunofluorescence. Confocal images were analyzed to determine intensity of staining in utero-placental compartments.

Results: In both experiments, ESR1 and ESR2 were expressed in fetal membranes (chorion or cho rioallantois), endometrial stroma (ES) and endometrial glands (EG), ESR1 was present in luminal epithelium (LE) and myometrium (Myo), and ESR2 was present in endometrial blood vessels (EBV). In Experiment 1, ESR1 expression was greater in LE and EG but was similar in ES and Myo in non-pregnant compared with pregnant ewes. ESR2 expression was similar in non-pregnant and pregnant compartments including LE, EG, ES, Myo, EBV and Myo blood vessels. In Experiment 2, ESR1 was expressed in LE, EG and Myo in NAT but was not detectable in NAT-ET, IVF and IVA groups; ESR2 was expressed in EG and EBV of NAT and NAT- ET but was not detected in IVF and IVA; expression of ESR1 and ESR2 was very low in ES of all groups.

Conclusion: These data demonstrate a differential localization pattern of ESR1 and ESR2 in uteroplacental compartments in early pregnancy compared with non-pregnant and after ART, indicating a specific expression depending on pregnancy stage and embryo origin.

Support: NIH grant 1R03HD076073-02 to LPR and ATGB.

P1-3: Expression of c-reactive protein is increased in placental tissue of women with pre-eclampsia L Best ${ }^{1}$, A Parisien $^{1}$, C Azure $^{1}$, B Davis ${ }^{2}$, C Anderson ${ }^{3}$

${ }^{1}$ Turtle Mountain Community College, Belcourt, ND, USA, ${ }^{2}$ University of North Dakota, Grand Forks, ND, USA, ${ }^{3}$ Ohio State University, Columbus, OH, USA

Objective: The etiology of the pregnancy complication, pre-eclampsia (PE), is unknown; but mul tiple lines of evidence implicate immunologic factors as important contributors. C-reactive protein (CRP) is a prominent component of the innate immune system; and we previously reported inherited genetic variants that increase the risk of $\mathrm{PE}$. Very recently the placenta has been found to express CRP and infusion of CRP into pregnant mice has recapitulated various features of PE. We sought to replicate some of these latter findings.

Methods: Placental tissue from 6 women with PE and 8 with normal pregnancies was obtained. RNA was extracted and cDNA produced using standard methods. Quantitative, real-time PCR using BioRad primers for human CRP and a standard "housekeeping" gene (GAPDH) was used to estimate placental expression of CRP (the Cq value) as a ratio of GAPDH expression (CRP/ GAPDH). Each expression run measured SYBR fluorescence in triplicate and Cq values were determined against a standard curve derived from BioRad template standards of known concentration. Student's $t$ test examined possible differences of mean CRP/GAPDH ratios. 
Results: There were a total of 22 triplicate runs of CRP/GAPDH expression. Intra-run CV's averaged $1.51 \%$. The mean (SD) CRP/GAPDH ratio was $1.03(0.024)$ and $1.14(0.060)$ for cases and controls respectively, $\mathrm{p}<0.001$

Conclusion: We show that CRP is expressed in placental tissue and that expression is greater relative to a standard reference gene among those with PE compared with normal pregnancies. Our findings are consistent with the single previous investigation of this question.

Support: NIH grant P20GM103442.

P1-4: High density lipoprotein (HDL) is the major provider of maternal cholesterol to the placenta and a dominant transporter of cholesterol from the placenta to the fetus in vivo AM Holme ${ }^{1}$, H Horne ${ }^{1}$, MCP Roland ${ }^{1}$, MB Holm ${ }^{1}$, TM Michelsen ${ }^{1}$, T Henriksen ${ }^{1}$ ${ }^{1}$ Oslo University Hospital, Oslo, Norway

Objective: Experimental evidence suggest that maternal HDL-cholesterol is taken up by placental trophoblastic cells on the maternal side and that fetal HDL takes up cholesterol from placental endothelium on the fetal side. A similar role of HDL in vivo would predict a net uptake of HDLcholesterol by the placenta on the maternal side and a net transfer of HDL-cholesterol from the umbilical circulation to the fetus. We tested this hypothesis by assessing the arteriovenous concentration differences on both sides of the human placenta in vivo.

Methods: Blood samples from 177 healthy women, with uncomplicated pregnancies, were obtained from the uterine vein and radial artery, and from the umbilical vein and artery at planned cesarean section. Paired arteriovenous concentration differences of HDL- and LDL-cholesterol on the maternal (uteroplacental) and fetal (umbilical) side of the placenta were assessed with Wilcoxon signed rank test and presented as median(IQR).

Results: In accordance with a placental uptake from the maternal circulation we found a significant uteroplacental arteriovenous difference of HDL-cholesterol $0.012(-0.007,0.037) \mathrm{mmol} / \mathrm{L}$ $(\mathrm{p} \leq 0.001)$, whereas the uteroplacental difference of LDL-cholesterol was not significant $(0.013$ $(-0.030,0.055) \mathrm{mmol} / \mathrm{L},(\mathrm{p}=0.07))$. At the fetal side of the placenta, both the umbilical venoarterial concentration difference of HDL-cholesterol $0.016(0.003,0.036) \mathrm{mmol} / \mathrm{L}$ and LDL-cholestero $0.012(-0.002,0.026) \mathrm{mmol} / \mathrm{L}$ was significant $(\mathrm{p}<0.001)$, indicating placental release/fetal uptake of both. When compared, both as direct values and as a fraction of the total cholesterol, the umbilical venoarterial difference of HDL-cholesterol was higher than LDL-cholesterol, $(\mathrm{p}<0.001)$.

Conclusion: Our human in vivo study indicates that HDL-cholesterol is the major provider of maternal cholesterol to the placenta. On the fetal side both umbilical HDL and LDL transport cholesterol from the placenta to the fetus, although HDL is quantitatively dominant.

Support: South-Eastern Norway Regional Health Authority Grants.

P1-5: The human in vivo uteroplacental uptake of glucose, triglyceride and cholesterol are correlated, but seem largely independent of placental weight

AM Holme ${ }^{1}$, MCP Roland ${ }^{1}$, MB Holm ${ }^{1}$, H Horne $^{1}$, TM Michelsen ${ }^{1}$, T Henriksen ${ }^{1}$

${ }^{1}$ University of Oslo, Oslo, Norway

Objective: Maternal metabolic factors are known to affect birth weight and fat accretion in the offspring. As some of these factors also affect placental weight, we hypothesized that they by modifying placental weight also modify nutrient transport capacity to the fetus. Following this hypothesis we expected the placental weight to be correlated to the uteroplacental uptake of different nutrients. Further we anticipated that per liter blood passing the placenta, the uteroplacental uptake of the individual nutrients would be correlated.

Methods: A cross-sectional in vivo study including 177 healthy women with uncomplicated pregnancies undergoing planned cesarean section. Blood samples were obtained from the uterine vein and the radial artery before uterine incision. They were analyzed for glucose, triglyceride, totalcholesterol, low- (LDL) and high-density lipoprotein (HDL)-cholesterol. Paired arteriovenous concentration differences on the maternal side (reflecting uteroplacental uptake) of the placenta were assessed with Wilcoxon sign rank tests and correlations were reported as Pearson's $r$ or Spearman's rho as appropriate.

Results: In our study placenta weight was correlated to maternal body mass index ( $\mathrm{r}=0.20, \mathrm{p}=0.012)$, gestational weight gain $(r=0.27, p \leq 0.001)$ and triglyceride $(r=0.28, p \leq 0.001)$. There was a negative correlation between placenta weight and HDL-cholesterol $(r=-0.23, p=0.003)$. The uteroplacental arteriovenous differences $(\mathrm{mmol} / \mathrm{L})$ of glucose, triglyceride, total-, LDL-, and HDL-cholesterol were all positively correlated, with Spearman's rho ranging from 0.207 to 0.552 and p-values $\leq 0.007$. In contrast to what was expected the placental weight was not correlated to the uteroplacental uptake $(\mathrm{mmol} / \mathrm{L})$ of glucose, HDL- and LDL-cholesterol. It was however associated with the uptake of triglyceride $(\mathrm{r}=0.16, \mathrm{p}=0.04)$ and total-cholesterol $(\mathrm{r}=0.17, \mathrm{p}=0.03)$

Conclusion: The placental efficiency of nutrient uptake seems universal rather than specific for certain nutrients, but we could not explain this by mere placental weight in this model. This may suggest differences of placental metabolic properties independent of placental weight.

Support: South-Eastern Norway Regional Health Authority Grants.

\section{P1-6: (Withdrawn)}

P1-7: Transcriptomic changes in the placenta in response to chronic maternal hypercortisolemia in late gestation

S Joseph ${ }^{1}$, EM Richards ${ }^{1}$, M Keller-Wood ${ }^{1}$

${ }^{1}$ University of Florida, Gainesville, FL, USA

In a sheep model of maternal stress, pregnant ewes were subjected to continuous cortisol infusion $(1 \mathrm{mg} / \mathrm{kg} /$ day) from 115 days of pregnancy, and euthanized at term. Placentomes were collected and RNA extracted from treated $(n=5)$ and untreated $(n=7)$ pregnancies. Microarray was performed on an ovine gene array (Agilent). Significantly changed genes were discovered using Limma package in $\mathrm{R}$, pathway inference was performed using Webgestalt and Cytoscape. 664 genes were differentially regulated, $(P \leq 0.05)$ of which 539 were downregulated and 125 upregulated. Gene ontology $(\mathrm{GO})$ analysis (Webgestalt) of these genes suggested 149 genes were stress related and 132 involved in small molecule metabolism. KEGG analysis (Webgestalt) showed downregulation of the insulin signaling pathway, as well as downregulation of glycolysis and fatty acid metabolism. Changes in glucose metabolism have also been indicated by transcriptomic analyses of the heart and skeletal muscle from these same fetuses, suggesting systemic effects of hypercortisolemia on metabolism. In similarly-treated fetuses, cortisol decreased serum fructose but failed to increase plasma glucose, despite relative maternal hyperglycemia, suggesting altered placental metabolism or transport of sugars. Transporters associated with proteins, sugars, and the inorganic metals, copper and zinc were downregulated too. Pathway inference also suggested structural changes in the placenta, for example 12 genes related to focal adhesion were downregulated. Expression of the steroid metabolic genes, HSD17B6 and androgen receptor were altered, along with HDAC's which modulate epigenetic changes in the placenta, and qPCR results validated the known downregulation of IGF2, a crucial imprinted gene that controls growth. Transcription factor analysis showed 151 of the genes contained an SP1 response element, 44, the Estrogen Related Receptor 1 (ERR1), but only 12 were glucocorticoid responsive (GR). These results suggest that maternal stress may alter placental glucose metabolism, and impair placental structure and function and in turn may be responsible for the altered metabolic profile in the fetuses. Support: National Institute of Health (HD057871)

P1-8: Peroxisome proliferator-activated receptor gamma (PPARG) and its effects on altitudeassociated fetal growth restriction and placental vascularization

CG Julian ${ }^{1}$, G Wolfson ${ }^{1}$, H Rathi ${ }^{2}$, RB Dodson ${ }^{1}$, DASchwartz ${ }^{1}$

${ }^{1}$ University of Colorado, Aurora, CO, USA, ${ }^{2}$ University of Colorado, Denver, CO, USA

High-altitude hypoxia impairs maternal vascular adaptation to pregnancy and reduces fetal growth. Our human studies demonstrate that maternal hypoxia represses the expression of peroxisome proliferator-activated receptor gamma (PPARG), a ligand-activated nuclear receptor central for energy homeostasis and vascular adaptation during pregnancy, as has been observed in fetal growth restriction (FGR) and preeclampsia.

Objective: To determine whether PPARG agonist (pioglitazone [PIO]) treatment during the pregnancy protects against altitude-associated FGR and to establish the effect of PIO treatment on placental vascularization.

Methods: Experiments were performed using four groups of C57/BL6 mice distinguished by control or PIO $(0.2 \%$ Actos, containing $0.05 \%$ pioglitazone $\mathrm{HCl})$ diet, and exposure to either normoxia $(\mathrm{Nx})$ or hypoxia $(\mathrm{Hx})$ during pregnancy (Control $+\mathrm{Nx}$, Control $+\mathrm{Hx}$, $\mathrm{PIO}+\mathrm{Nx}$ and $\mathrm{PIO}+\mathrm{Hx}$ ). Assigned diets began at the time of mating and were sustained throughout the protocol. For the $\mathrm{Nx}$ and $\mathrm{Hx}$ exposures were placed in a hyperbaric $(760 \mathrm{mmHg})$ or hypobaric chamber $(375 \mathrm{mmHg})$ from gestational day (GD) 14.5 to 17.5 . On GD 17.5 we measured fetal weight, crown-rump length, biparietal diameter and placental weight. Placental tissue was harvested for CD34 staining and vascular density measurements. Fetal sex was determined by PCR.

Results: Hx reduced fetal weight (Control and PIO) and increased placental weight (Control), but had no effect on placental vascularity. PIO (1) reduced fetal weight, increased the number of fetal reabsorptions and impaired placental vascularity (vessel number and density) under $\mathrm{Nx}$ and $\mathrm{Hx}$ conditions, and (2) lowered placental weight in Hx animals.

Conclusion: PIO treatment across the entire pregnancy aggravates altitude-associated fetal growth restriction and impairs placental vascular density. Follow-up experiments will test whether PIO treatment exclusively during the hypoxic exposure effectively protects fetal growth. Support: 5 K12 HD057022-07 and AHA 15BGIA25680022.

P1-9: Preconceptional lipid-based micronutrient supplementation reduced circulating branched chain amino acids in guatemalan women who are overweight or obese at 12 weeks gestation

M Kerns ${ }^{1}$, P Baker 2 , J Kemp 2 , S Morrison ${ }^{3}$, J Westcott ${ }^{2}$, J Friedman², A Garces ${ }^{4}$, L Figueroa ${ }^{5}$, $\mathrm{M} \mathrm{Hambidge}^{2}$, N Krebs ${ }^{2}$, S Borengasser ${ }^{2}$

${ }^{1}$ University of Colorado, Denver, CO, USA; ${ }^{2}$ University of Colorado, Aurora, CO, USA; ${ }^{3}$ Ben Gurion University of Negev, Beer-Sheva, Israel; ${ }^{4}$ Institute of Nutrition of Central America and Panama, Guatemala City, Guatemala

Childhood obesity is a global health concern. Estimates project 60 million preschool- aged children worldwide will be overweight or obese (OW/OB) by 2020. Recent reports suggest that low- and middle-income countries are suffering from a "double burden" of undernutrition (growth stunting) and over nutrition (obesity). DOHaD has demonstrated in utero exposure to maternal under- or over nutrition contributes to detrimental growth and metabolic outcomes in later life. Elevated branched chain amino acids (BCAAs: Valine, Leucine, and Isoleucine) are well-established biomarkers of insulin resistance (IR). The objective of this pilot study was to examine if circulating BCAA levels can serve as biomarkers for obesity-associated IR and test whether a daily lipid-based micronutrient supplement (LNS) can improve metabolic status in Guatemalan women experiencing stunted growth and/or obesity during early pregnancy. Using an ongoing RCT (Women First) we studied women who were normal weight $(\mathrm{NW}, \mathrm{BMI}=20.1-24.1)$ or $\mathrm{OW} / \mathrm{OB}(\mathrm{BMI}=25.6-31.9)$, and divided into two arms (n $=9-10$ /group): those who received daily LNS $\geq 3$ mos prior to conception through 12 wk gestation (+LNS), or no LNS (-LNS). LNS compliance was $\geq 80 \%$ and carefully monitored by on-site personnel via biweekly collection of empty satchels, compliance calendars, and random audits. Circulating BCAAs were elevated in OW/OB-LNS at 12 wk compared to NW-LNS $(\mathrm{p}=0.001)$. OW/OB+LNS had reduced BCAAs compared to OW/OB-LNS ( $\mathrm{p}=0.007$ ), no change in NW+LNS vs NW-LNS, suggesting LNS may improve metabolic status in OW/OB mothers and reduce detrimental fetal exposures. This study suggests that preconception LNS may impact maternal metabolic health.

Support: Bill \& Melinda Gates Foundation OPPI 055867, NIH \& ODS \#U10 HD076474, \& NIH 1 K01 DK109077-01.

P1-10: Higher levels of oxidative stress and brain derived neurotrophic factor in the central maternal region in the placentas of mothers with preeclampsia

D Sundrani ${ }^{1}$, A Sahay ${ }^{1}$, G Wagh ${ }^{2}$, S Mehendale ${ }^{2}$, C Yajnik ${ }^{3}$, S Joshi

${ }^{1}$ Bharati Vidyapeeth University, Pune, Maharashtra, India. ${ }^{2}$ Bharati Medical College and Hospital, Pune, Maharashtra, India. ${ }^{3} \mathrm{KEM}$ Hospital and Research Centre, Pune, Maharashtra, India Objective: To examine the regional differences in placental oxidative stress marker malondialdehyde (MDA) and brain derived neurotrophic factor (BDNF) in various regions of the normotensive control (NC) placenta and placenta from a woman with preeclampsia (PE)

Methods: A total of $50 \mathrm{NC}$ women and 47 women with PE (21 delivering at term and 26 delivering preterm) were recruited for this study. Placenta was collected from four different regions i.e. central maternal (CM), central fetal (CF), peripheral maternal (PM) and peripheral fetal (PF).

Results: The levels of MDA were higher in all regions of the placenta from term and preterm PE group ( $<<0.01$ for all) as compared to NC group. In the preterm PE group, the MDA levels were higher in central maternal $(\mathrm{p}<0.05)$ as compared to central fetal region. The levels of BDNF were 
higher in CF region as compared to CM $(\mathrm{p}<0.01), \mathrm{PM}(\mathrm{p}<0.01)$ and $\mathrm{PF}(\mathrm{p}<0.05)$ regions of the placenta in the control group. The BDNF levels in the CM region $(\mathrm{p}<0.05)$ were higher in the preterm preeclampsia group as compared to the normotensive control group.

Conclusion: Differential oxidative stress levels exist in the central maternal and central fetal placental regions of women with preterm preeclampsia. This pattern was not detected among normotensive women or women with preeclampsia who delivered at term. The higher levels of oxidative stress may be due to hypoxia and inflammation which is involved in the pathogenesis of preterm preeclampsia. Higher levels of BDNF and MDA in the central maternal region suggest that this region may play an important role in influencing the placental functions.

Support: Indian Council of Medical Research (ICMR), India.

P1-11: The role of maternal dietary melatonin supplementation on binucleate cell number, size, and percentage in the ovine placentome

AR Tanner ${ }^{1}, V_{\text {Valkenov }}^{1}$, A Reyaz ${ }^{1}$, CO Lemley ${ }^{2}$, KA Vonnahme ${ }^{1}$

${ }^{1}$ North Dakota State University, Fargo, ND, USA. ${ }^{2}$ Mississippi State University, MS, USA

Melatonin supplementation has been previously demonstrated to increase umbilical arterial blood flow and potentially decrease oxidative stress in placental vascular beds. Additionally, previous research has suggested that trophoblast giant cells (binucleate cells; BNC) cells in the placenta may be secreting vasoactive factors. The objective of this study was to understand the therapeutic role of dietary melatonin supplementation in an ovine model of intrauterine growth restriction (IUGR) on binucleate cell measurements. From d 50 to 130 of gestation, ewes arranged in a $2 \times 2$ factorial design were fed either an adequate $(100 \%$ National Research Council; $n=15)$ or restricted $(60 \%$ Nationa Research Council; $\mathrm{n}=15)$ diet and supplemented with $5 \mathrm{mg}$ of melatonin $(\mathrm{n}=16)$ or no melatonin $(\mathrm{n}=14)$. At d 130, placentomes were collected and fixed in $10 \%$ formalin solution. Tissues were sectioned and stained with $10 \%$ normal goat serum, biotintylated lectin DBA, Texas red avidin, fluorescein BSL1, and DAPI mounting media to visualize the BNCs. BNC number and BNC size were determined using imaging analysis software. There was no impact of maternal nutritional plane on any binucleate cell measurements $(\mathrm{P}>0.5)$. Melatonin supplemented ewes tended $(\mathrm{P}=0.12)$ to have larger binucleate cells $\left(481.85\right.$ vs. $\left.321.56 \pm 73.35 \mu \mathrm{m}^{2}\right)$ than their control counterparts. Ewes treated with melatonin had similar $(\mathrm{P}=0.16)$ percentage of binucleate cells $(4.7 \%$ vs. $3.6 \% \pm 0.55)$ in cotyledonary tissue compared with control ewes. BNC number was not influenced $(\mathrm{P}>0.5)$ by melatonin supplementation ( 55.1 vs. $52.9 \pm 3.94)$. While umbilical blood flow and vasoreactivity were altered due to melatonin feeding, impacts of melatonin on BNCs within the placentome appear to be limited. The specific factors that BNCs may be secreting have yet to be elucidated and merit further study.

P1-12: The capacity for oxidation of long-chain fatty acids in human cytotrophoblast is dimin ished with syncytialization

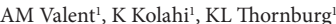

${ }^{1}$ Oregon Health \& Science University, Portland, OR, USA.

Objective: To test the hypothesis that the capacity of human cytotrophoblast (CTB) to oxidize fatty acids is unchanged by syncytialization.

Methods: Human CTB were isolated from normal term human placentas delivered via cesarean section and cultured in vitro. Measurement of intact cellular respiration was performed using the Seahorse XFe 96 analyzer of CTB at 8 hours and cultured, differentiated syncytialized cytotrophoblast (SCT) at 72 hours. Cells were treated with Palmitate $(250 \mu \mathrm{M})$, Oleate $(250 \mu \mathrm{M})$ or vehicle bovine serum albumin $(40 \mu \mathrm{M})$ immediately prior to assay measurements. Respiration was measured under basal conditions, in the presence of the mitochondrial ATP synthase inhibitor Oligomycin, the mitochondrial uncoupling compound carbonylcyanide-4-trifluorometh-oxyphenylhy drazone, and the respiratory chain inhibitors Rotenone/Antimycin A. Oxygen consumption rates were normalized to total DNA content. Paired t-test was used to compare oxygen consumption rates between CTB and SCT.

Results: Palmitate and Oleate administration invoked a significant increase in oxygen consumption rate in CTB compared to SCT. Under maximal uncoupling conditions carbonylcyanide-4-trifluorometh-oxyphenylhydrazone, CTB oxygen consumption rate is $60 \%$ higher than for SCT. Oxygen consumption due to ATP production is significantly higher in CTB compared to SCT after both Palmitate and Oleate administration.

Conclusion: Contrary to our hypothesis, СTB have a higher capacity to metabolize fatty acids. Because CTB have a greater oxygen consumption rate for ATP production and we have previously shown that CTB metabolizes FA to a greater degree than SCT, we predict CTB have greater intracellular ATP concentrations.

Support: NICHD P01HD034430.

P1-13: Leptin receptor expression during the ovine ovarian cycle and late pregnancy: role of leptin and adiponectin on uterine artery endothelial cells angiogenic responses

VE Vargas ${ }^{1,2}$, R Villalon Landeros ${ }^{1}$, GE Lopez ${ }^{1}$, J Zheng ${ }^{1}$, RR Magness ${ }^{1,2}$

'University of South Florida, Tampa FL, USA, ${ }^{2}$ University of Wisconsin, Madison, WI, USA

Objective: Pregnancy is characterized by elevated uterine blood flow (UBF) mediated in part by angiogenesis. Leptin and Adiponectin (ADN) regulate obesity and reproductive processes. How ever, little is known concerning the role(s) of Leptin and ADN in uterine angiogenesis and if this is pregnancy specific. We hypothesized that full-length ADN (fADN), globular ADN (gADN), and/ or Leptin treatment will differentially modulate cell proliferation in uterine artery endothelial cells (UAECs) derived from nonpregnant (NP-Luteal and Follicular) vs. late pregnant (P-) ewes.

Methods: Leptin receptor protein was evaluated in passage $4 \mathrm{NP}$ and P-UAECs. Ovine UAECs were also obtained from NP, $(n=4)$, and $\mathrm{P}(120-130 \mathrm{~d} ; \mathrm{n}=4)$ sheep and were treated with vehicle (control), fADN $(5 \mu \mathrm{g} / \mathrm{ml}), \mathrm{gADN}(1-20 \mu \mathrm{g} / \mathrm{ml})$ or Leptin $(1-1000 \mathrm{ng} / \mathrm{ml})$ for $24 \mathrm{hr}$ and proliferation measured using 5-ethynyl-2-deoxyuridine assays.

Results: Both $\mathrm{OB}-\mathrm{Ra} / \mathrm{OB}-\mathrm{Rb}$ proteins were expressed with similar levels between groups Although fADN did not alter UAEC proliferation in any group, gADN inhibited (-53\%) P-UAEC proliferation $(0.47 \pm 0.01$ fold; $\mathrm{P}<0.05)$, but not Luteal or Follicular NP-UAECs $(0.98 \pm 0.04$ fold $)$. Leptin treatment did not alter Luteal phase UAEC proliferation $(0.80 \pm 0.20$ fold); however, it promoted proliferation in Follicular NP-UAECs $(1.90 \pm 0.24$ fold; $\mathrm{P}<0.01)$ and P-UAECs $(1.53 \pm 0.22$ fold; $\mathrm{P}<0.01)$.
Conclusion: Leptin receptors were similar between groups. fADN did not modulate proliferation in the NP- and P-UAECs. gADN anti-angiogenic effect was pregnancy specific, i.e. not seen in the NP-UAECs. Leptin-stimulated proliferation responses are seen at nanomolar levels and but only in the UAECs from in the two high estrogen physiologic states (follicular phase and pregnancy). Therefore leptin may play an important angiogenic role in preparation for the increase UBF during the periovulatory and possibly pregnancy period. However, gADN/Leptin may regulate uterine artery angiogenesis in preparation for the increase blood flow demands of the growing fetus. Support: NIH-HL49210, HD38843, HL87144, HL117341.

P1-14: Realimentation in ewes during late gestation alters binucleate cell number in the placentome

MA Vasquez, A Reyaz, JS Caton, KA Vonnahme

Department of Animal Sciences, North Dakota State University, Fargo, NC, USA

To determine the effects of maternal plane of nutrition during mid and/or late gestation on binucleate cell (BNC) numbers, size, and percentage of the fetal-maternal interface, pregnant ewe lambs $(\mathrm{n}=32)$ were assigned to 1 of 4 treatments arranged in a $2 \times 2$ factorial arrangement: nutritional level [100\% (control; CON) or $60 \%$ (restricted; RES) of NRC recommendations] fed at different times of gestation [d 50 to 90 (Mid) or d 91 to 130 (Late)]. At day $130 \pm 1$ of gestation ewes were euthanized and placentomes were collected and fixed in formalin solution. Tissue sections were stained with biotinylated DBA lectin, texas red avidin, and fluorescein BSL I lectin, all diluted in $10 \%$ normal goat serum. DAPI mounting media was used to visualize the BNCs. Maternal plane of nutrition during Late gestation affected fetal weight with $\mathrm{CON}$ ewes having heavier lambs $(\mathrm{P}=0.01)$ than RES ewes ( 4.13 vs. $3.75 \pm 0.10 \mathrm{~kg}$ ). There was no effect $(\mathrm{P}>0.39)$ of diet on any measure of placental weight or size. There was no effect $(\mathrm{P}>0.27)$ of maternal plane of nutrition on BNC size Binucleate cell number per tissue area was decreased $(\mathrm{P}<0.01)$ in RES-CON ewes compared to all other ewes (138.3 vs. $213.9,196.5,196.2 \pm 15.6$ for RES-CON vs RES-RES, CON-RES, CON-CON, respectively). Percentage of $\mathrm{BNC}$ area per tissue area tended to be reduced $(\mathrm{P}=0.10)$ in RES-CON ewes compared to all other treatments. It appears that maternal nutritional plane may impact BNC development throughout gestation as increases in intake results in reduction in the BNC development. Perhaps metabolic cues from the dam are altering the timing and development of BNCs.

P1-15: A preliminary trial of pregnancy outcomes and potential sex selection following embryo selection by specific gravity

CE Wessels ${ }^{1}$, L Penrose ${ }^{1}$, S Prien ${ }^{1}$

${ }^{1}$ Texas Tech University, Lubbock, TX, USA

Objective: Given the improvement in embryo culture techniques, there is a growing consensus that most, if not all (Assisted Reproductive Technologies) ART should be done by single embryo transfer (SET). However, to do so, an improved means of embryo selection must be developed. This lab has previously described a non-invasive means of embryo selection based on specific gravity. The current study presents outcome data from the first preliminary pregnancy trial in an animal model. Methods: Cryopreserved sheep blastocysts were thawed and assessed for viability based on morphological assessment. Thawed blastocysts were then assessed using the Noninvasive Embryo Assessment Technique (NEAT) based on an estimation of embryo weight (EEW). Blastocyst pairs of similar weights were transferred into recipient ewes $(\mathrm{N}=15)$. Pregnancy was first confirmed by DG29 $9^{\text {ma }}$ pregnancy test Twin versus singleton pregnancies were confirmed after lambing. Both initial pregnancy outcomes and live lambs were correlated back to embryo weight to establish if EEW correlated pregnancy.

Conclusion: A total of $6 / 15$ ewes ( $40 \%$ ) conceived after embryo transfer. In this small preliminary study, there was a correlation between EEW in sheep that conceived verses those who did not $(\mathrm{P}<0.046)$. Further, all pregnancies continued to term without loss $(\mathrm{P}<0.046)$. Too few singleton pregnancies occurred to correlate birth outcomes (singleton vs. twin) to EEW. Previous studies have suggested differences in the EEW were due to differences in embryo composition, in some cases due to damage to cellular membranes. The current study demonstrated a difference in the EEW and pregnancy outcomes in cryopreserved embryos. While it is unclear if the differences seen were due to membrane integrity or cellular composition, the data continues to suggest the usefulness of EEW in embryo selection.

\section{POSTER SESSION 2 - FETUS, NEONATE AND CHILD}

P2-1: Chronic maternal hypercortisolemia in late gestation increases fetal P-R interval and reduces heart rate during delivery

A Antolic ${ }^{1}$, C Wood ${ }^{1}$, M Keller-Wood

${ }^{1}$ University of Florida, Gainesville, FL, USA

In previous studies in an ovine model, we found that maternal hypercortisolemia increased perinatal stillbirth, thickening of the cardiac left ventricle and septum, Purkinje fiber apoptosis, and altered the cardiac transcriptome related to metabolism and ion channels. We tested the hypothesis that cortisol altered fetal mean aortic pressure (MAP), heart rate (HR), pulse height $(\mathrm{PH})$, and/or ECG during maternal cortisol infusion. Four ewes with singleton fetuses were chronically infused with $1 \mathrm{mg} / \mathrm{kg} / \mathrm{d}$ cortisol (CORT) from 115 days of gestation to term. Five ewes were not treated. Radiotelemetry devices were implanted into the fetuses at $119 \pm 1 \mathrm{~d}$ for continuous monitoring of ECG, HR, and aortic and amniotic fluid pressures through delivery. We used DSI software to analyze parameters of the ECG, HR, PH, and MAP using $1 \mathrm{~h}$ means for the first $24 \mathrm{~h}$ following surgery and then every $6 \mathrm{~h}$ for the following 4 days to test for effects of CORT on recovery from surgery; subsequently we analyzed $1 \mathrm{~h}$ means for the $14 \mathrm{~d}$ before birth or the last $24 \mathrm{~h}$, and $10 \mathrm{~min}$ means during the final hour before delivery. Data were analyzed by two-way ANOVA corrected for repeated measures across time, with comparison of means by t-test with Bonferroni adjustment. During recovery from surgery, CORT altered the time course of the MAP, although the MAP overall was not different in the CORT fetuses. CORT significantly elongated the P-R interval after surgery, and significantly lengthened the $\mathrm{P}$ and $\mathrm{P}-\mathrm{R}$ intervals in the last $24 \mathrm{~h}$ before birth. In the hour before birth CORT significantly reduced HR and the ratio of Tpe (interval from the peak to the end of the T wave) to the corrected QT interval was also significantly increased. These results suggest that CORT induces pathophysiological alterations in the cardiac conduction pathway, becoming most apparent during the perinatal period. These changes may make the fetus susceptible to increased risk for arrhythmias and cardiac failure during stress of labor and delivery.

Support: NIH HD057871; AHA 14GRNT20420048. 


\section{Abstracts}

P2-2: Ovine uterine space restriction causes dysregulation of the renin angiotensin system in fetal kidneys

AS Bauer ${ }^{1}$, RA Kranch-Shorthouse ${ }^{1,2}$, GE Lopez ${ }^{1}$, RR Magness ${ }^{1}$, JL Segar ${ }^{3}$, SE Blohowiak ${ }^{1}$, PJ Kling ${ }^{1}$ University of Wisconsin-Madison, Madison, WI, USA. ${ }^{2}$ University of Arizona, Tucson, AZ, USA. University of Wisconsin-Madison, Madison, WI, USA, ${ }^{3}$ University of Iowa, Iowa City, IA, USA

Objective: To test the hypothesis that uterine space restriction (USR) increases angiotensin receptor expression and alters intravascular fluid and electrolyte homeostasis in fetal ovine kidneys.

Methods: Multiparous ewes with reduction in placental attachment sites and multiple fetuses (USR) were compared to non-space restricted (NSR) singleton controls. Fetal kidneys and blood were collected on either gestational day (GD) 120 or GD130 (term=GD147). Fetal blood was analyzed for plasma chemistry and renin activity. Kidneys were analyzed for Angiotensin $\mathrm{II}_{1-8}$ Receptor Type 1 $\left(\mathrm{AT}_{1} \mathrm{R}\right)$, Angiotensin $\mathrm{II}_{1-8}$ Receptor Type 2 ( $\left.\mathrm{AT}_{2} \mathrm{R}\right)$, and Angiotensin $\mathrm{II}_{1-7}$ Mas Receptor (MASR) by Western Blot and immunohistochemistry.

Results: Expression of AT, R, AT R, and MASR increased in USR kidneys between GD120 and $\mathrm{GD} 130(P<0.05)$. Expression of $\mathrm{AT}_{1} \mathrm{R}$ and $\mathrm{AT}_{2} \mathrm{R}$ was localized in a more homogeneous pattern, either transmembranous or intracytoplasmic in the proximal and distal tubules while MASR localized intranuclear in a discrete punctate vesicular pattern, in proximal and distal tubules, macula densa and glomeruli. Plasma renin activity (PRA) was decreased in USR by GD130 $(P<0.03)$ and was inversely related to plasma osmolarity $(P<0.02)$

Conclusion: USR upregulated renal angiotensin receptor expression that was more pronounced as gestation progressed. $\mathrm{AT}_{1} \mathrm{R}$ and $\mathrm{AT}_{2} \mathrm{R}$ play roles in renal development, but little was previously known about the MASR in renal development. Because MASR contains a nuclear localization sequence and produces nitric oxide in the nuclei in adult kidneys, it also may do so in development. Relationships between PRA, osmolarity, and angiotensin receptors support a role for reninangiotensin system (RAS) regulation of fetal intravascular fluid and electrolyte homeostasis. USR dysregulation of RAS may contribute to hypertension later in life.

Support: NIH-HL87144, HL49210, HD 38843, Meriter Foundation, UW Dept. Pediatrics, UA Grad Research Assistantship, Nutr Sciences.

\section{P2-3: The effect of pulsatile hyperglycemia on insulin secretion in IUGR fetal sheep.}

B Boehmer ${ }^{1}$, L Brown ${ }^{1}$, S Wesolowski ${ }^{1}$, W Ha ${ }^{1} y$, B Strahan ${ }^{1}$, P Rozance ${ }^{1}$

${ }^{1}$ University of Colorado, Aurora, CO, USA

Normal ovine fetuses respond to repeated pulsatile hyperglycemia (PHG) with increased glucose stimulated insulin secretion (GSIS). The objective of these studies was to evaluate the effect of PHG ( $\mathrm{n}=7$ fetuses) or saline infusions (SAL; $\mathrm{n}=7$ fetuses) on insulin secretion in twin fetal sheep from placental insufficiency IUGR pregnancies. Five to $7 \mathrm{~d}$ after surgery to place indwelling intravenous and arterial catheters ( 119 dGA), each twin fetus was randomly assigned to either PHG or SAL treatments. Fetal PHG was induced by a constant, basal infusions of 33\% dextrose (D33; w/v) into the fetus, plus three $60 \mathrm{~min}$ pulses each day at 0900,1500 , and $2100 \mathrm{hrs}$. Basal infusions and pulses targeted an increase in fetal glucose concentrations of $20 \%$ and $80 \%$, respectively and were continued for $7 \mathrm{~d}$. GSIS was measured on the final study day by fetal square wave hyperglycemic clamp. Blood samples were collected daily before (PRE) and after (POST) $60 \mathrm{~min}$ pulses and regularly during GSIS (-15 to $90 \mathrm{~min}$; glucose clamp initiated at $0 \mathrm{~min}$ ) for measurement of plasma glucose and insulin concentrations. Baseline ( -5 to $-15 \mathrm{~min}$ ), early (5 to $45 \mathrm{~min}$ ), and late (60 to $90 \mathrm{~min}$ ) clamp phases were used to describe changes in GSIS. Insulin mRNA was analyzed by qPCR. Daily basal plasma glucose concentrations were $18 \%$ greater $(P<0.001)$ in PHG than in SAL fetuses and increased $76 \%(\mathrm{P}<0.001)$ during POST compared with PRE. Plasma insulin concentrations did not differ $(P=0.48)$ between PHG and SAL fetuses during the $7 \mathrm{~d}$ infusions. Although GSIS occurred in both treatment groups $(P<0.001)$, neither early and late phase plasma insulin concentrations nor pancreatic insulin mRNA abundance differed $(P \geq 0.12)$ between PHG and SAL fetuses. These results indicate that IUGR fetuses may have a reduced insulin response to varying glycemic patterns in contrast to normal fetuses, consistent with blunted GSIS response in IUGR fetuses under basal conditions.

Support: NIH R01 DK088139.

P2-4: Impacts of beta agonists and pro-inflammatory cytokines on basal and insulin-stimulated glucose metabolism in rat soleus

CN Cadaret ${ }^{1}$, KA Beede ${ }^{1}$, HE Riley ${ }^{1}$, DT Yates

${ }^{1}$ University of Nebraska-Lincoln, Lincoln, NE USA

Recent studies suggest that catecholamines and pro-inflammatory cytokines help regulate muscle growth and metabolism at sub-stress levels. Our objective was to determine acute effects of $\beta 1$ and $\beta 2$ adrenergic agonists, TNF $\alpha$, and IL- 6 on muscle glucose uptake and oxidation under basal and insulin-stimulated conditions. Soleus muscles from adult Sprague-Dawley rats were separated into $25-45 \mathrm{mg}$ strips and incubated in KHB spiked with insulin, ractopamine ( $\beta 1$ agonist), zilpaterol ( $\beta 2$ agonist), TNF $\alpha$, or IL-6. Glucose uptake was determined from cellular accumulation of $\left[{ }^{3} \mathrm{H}\right]$-2-deoxyglucose after $20 \mathrm{~min}$. Glucose oxidation of $\left[{ }^{14} \mathrm{C}\right.$-U $]$-glucose was determined after $2 \mathrm{~h}$. Phospho-Akt/ total Akt (p-Akt/Akt) was determined after 1h. Compared to muscle incubated in un-spiked (basal) media, incubation with insulin increased $(\mathrm{P}<0.05)$ glucose uptake by $\sim 47 \%$, oxidation by $\sim 32 \%$, and p-Akt/Akt by $\sim 238 \%$. Muscle incubated with $\beta 2$ agonist exhibited $\sim 20 \%$ less $(\mathrm{P}<0.05)$ glucose uptake but $\sim 32 \%$ greater $(\mathrm{P}<0.05)$ oxidation than basal. Moreover, $\beta 2$ agonist + insulin increased $(\mathrm{P}<0.05)$ glucose oxidation and p-Akt/Akt over insulin alone. $\beta 1$ agonist incubations did not differ from basal for any output. Likewise, $\beta 1$ agonist+insulin incubations did not differ from insulin alone. Glucose oxidation was $\sim 23 \%$ and $\sim 33 \%$ greater $(\mathrm{P}<0.05)$, respectively, in TNF $\alpha$ and IL- 6 incubations compared to basal, yet uptake and p-Akt/Akt did not differ. Glucose uptake, oxidation, and p-Akt/Akt were similar among TNF $\alpha+$ insulin, IL-6+insulin, and insulin alone. In addition, glucose oxidation in TNF $\alpha+$ +insulin and IL- $6+$ insulin incubations did not differ from TNF $\alpha$ or IL-6 alone. These results show that acute $\beta 2$ stimulation had opposite effects on muscle glucose uptake and oxidation, and that acute $\beta 1$ stimulation had no evident metabolic impact. Moreover, $\beta 2$ stimulation was synergistic with insulin, as glucose oxidation and Akt phosphorylation were greater with both agents together than either individually. Lastly, acute cytokine stimulation increased glucose oxidation independently of insulin or Akt phosphorylation. Together, our findings demonstrate that adrenergic and inflammatory mediators can have insulin-associated or insulin-independent effects on glucose metabolism and that these effects may differ for glucose uptake and oxidation.
P2-5: Thyroid hormone regulates VEGF expression in the near term sheep heart NN Chattergoon ${ }^{1}$, S Louey ${ }^{1}$, GD Giraud ${ }^{1,2}$, KL Thornburg $^{1,}$

${ }^{1}$ Oregon Health and Science University, Portland, OR; ${ }^{2}$ Portland VA Health Care System, Portland, OR, USA

Objective: The fetal left ventricle (LV) must be prepared for a near doubling of work load at birth, including having an adequate vascular tree to supply the muscle itself. Cardiac exposure to thyroid hormone $\left(\mathrm{T}_{3}\right) 15$ days prior to the normal prepartum surge promotes early maturation of cardiomyocytes. Given that $\mathrm{T}$ promotes postnatal capillary growth in adult rodents, we hypothesized that it also stimulates new vessel growth in the fetal heart. We also investigated the angiogenic effects of IGF-1 which stimulates cardiomyocyte proliferation; we tested whether it also stimulates increases in myocardial vascular growth.

Methods: Fetal sheep were instrumented at 120 days of gestation (dGA, term 145dGA) and studied from 125d GA. Study groups (n=8/group) were: 1) $\mathrm{T}_{3}(54 \mu \mathrm{g} / \mathrm{d}), 2$ ), Long R3 IGF-1 (IGF-1, $715 \mu \mathrm{g} / \mathrm{d})$, 3) Thyroidectomized (TX), 4) $\mathrm{T}$ combined with IGF-1 (T +IGF-1), and 5) control. At 130dGA, animals were euthanized and a section of fetal midwall LV was snap frozen for molecular analysis. Expression levels of vascular factors were determined by qPCR and normalized against $\beta$-actin levels. Significance following ANOVA and post-testing was set at $\mathrm{p}<0.05$.

Results: Compared to controls, $\mathrm{T}_{3}$ stimulated increases in HIF-1a (+100\%), VEGF $(+40 \%)$, and VEGF-R2 (+25\%); these levels were lower in thyroidectomized animals (HIF-1a: -175\%; VEGF: $-70 \%$; VEGF-R1: - $80 \%$; VEGF-R2: $-38 \%$ ) vs. $\mathrm{T}_{3}$-infused levels. Expression of these genes was not changed in IGF-1-infused animals compared to controls or $\mathrm{T}_{3}$. However, the combined treatment of $\mathrm{T}_{3}+\mathrm{IGF}-1$ suppressed expression of HIF-1a, VEGF and VEGF-R1 vs. $\mathrm{T}_{3}$ or IGF-1 alone.

Conclusion: Expression of VEGF-related genes in the near term fetal heart is regulated by circulating levels of $\mathrm{T}_{3}$ but not IGF-1. Interactions between $\mathrm{T}_{3}$ and IGF-1 signaling suppress vascular growth genes when both hormone levels are elevated simultaneously. The normal increase in $\mathrm{T}_{3}$ levels at term is likely to prepare the myocardium for increased loading conditions at birth by stimulating myocardial vascular growth.

Support: NHLBLI-K01HL118677

P2-6: Hypoxia induced expression of PCK1 and PGC1A and refractive AMPK activation in hepatocytes from sheep with intrauterine growth restriction

SL Coates ${ }^{1}$, LD Brown ${ }^{1}$, PJ Rozance ${ }^{1}$, SR Wesolowski ${ }^{1}$

${ }^{1}$ University of Colorado, Aurora, CO, USA

Objective: To understand the role of hypoxia in the activation of fetal hepatic glucose production, we used isolated hepatocytes from control (CON) and intrauterine growth restricted (IUGR) fetal sheep and measured 1) glucose production rate, and 2) effect of hypoxia exposure on expression of gluconeogenic genes (PCK1, PGC1A) and the phosphorylation of stress response protein (AMPK) and insulin signaling proteins (AKT, FOXO1).

Methods: Primary hepatocytes were isolated from CON $(n=5-6)$ and IUGR $(n=8-10)$ fetal sheep. Glucose production was measured after $24 \mathrm{~h}$ treatment in glucose-free media containing substrates

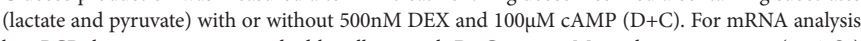
by $q$ PCR, hepatocytes were studied basally or with $\mathrm{D}+\mathrm{C}$ or $100 \mathrm{nM}$ insulin in normoxia $(21 \% \mathrm{O})$ or hypoxia $\left(3 \% \mathrm{O}_{2}\right)$ for 4 or $24 \mathrm{~h}$. For protein analysis by western blot, hepatocytes were treated with insulin $(0,1,10 \mathrm{nM})$ for $15 \mathrm{~min}$ following $4 \mathrm{~h}$ of normoxia or hypoxia. Data were analyzed by ANOVA. Results: IUGR hepatocytes had higher glucose production in response to $\mathrm{D}+\mathrm{C}$ compared to CON $(\mathrm{P}<0.05)$. In CON and IUGR cells, hypoxia for $4 \mathrm{~h}$ increased PCK1 and PGC1A $(\mathrm{P}<0.005)$. 4h of $\mathrm{D}+\mathrm{C}$ increased $P G C 1 A(\mathrm{P}<0.001) .4 \mathrm{~h}$ of insulin had no effect on expression of $P C K 1$ but decreased $P G C 1 A(\mathrm{P}<0.001)$. In CON and IUGR cells, hypoxia for $24 \mathrm{~h}$ increased PGC1A $(\mathrm{P}<0.001)$ but had no effect on PCK1. 24h of $\mathrm{D}+\mathrm{C}$ increased PCK1 and PGC1A (P<0.005). 24h of insulin decreased $P G C 1 A(\mathrm{P}<0.05)$ in normoxia but not hypoxia. Hypoxia for $4 \mathrm{~h}$ increased $\mathrm{P}-\mathrm{AMPK}$ in $\mathrm{CON}(\mathrm{P}<0.05)$ but not in IUGR. Insulin increased P-AKT and P-FOXO1 in CON and IUGR $(\mathrm{P}<0.001)$. P-AKT was further increased by hypoxia in IUGR $(\mathrm{P}<0.05)$.

Conclusion: We speculate that hypoxia in IUGR may contribute to increased glucose production through increased PCK1 and PGC1A and is potentiated by decreased P-AMPK, which may normally suppress glucose production. Further, although P-AKT and P-FOXO1 levels are maintained in hypoxia, the gluconeogenic gene PGC1A was not decreased with insulin during hypoxia exposure. Thus, impaired AMPK signaling along with upregulated PCK1 and PGC1A may provide a link to persistently increased hepatic glucose production in IUGR fetuses with hypoxia.

Support: NIH R03-DK102972.

P2-7: Combined treatment with L-citrulline and tetrahydrobiopterin $\left(\mathrm{BH}_{4}\right)$ synergistically improves nitric oxide (NO) signalling in hypoxic piglet pulmonary arterial endothelial cells (PAECs)

CD Fike ${ }^{1}$, A Dikalova $^{2}$, Y Zhang ${ }^{1}$, JL Aschner ${ }^{3}$

${ }^{1}$ University of Utah, Salt Lake City, UT, USA, ${ }^{2}$ Vanderbilt University, Nashville, TN, USA, ${ }^{3}$ Albert Einstein College of Medicine and the Children's Hospital at Montefiore, New York, NY, USA

Objective: Impairments in NO signalling, including endothelial nitric oxide synthase (eNOS) uncoupling, contribute to chronic hypoxia-induced pulmonary hypertension. When eNOS is uncoupled, eNOS activation generates superoxide instead of NO. We have previously shown that the L-arginine-NO precursor, L-citrulline, improves NO production by re-coupling eNOS in hypoxic PAEC. $\mathrm{BH}_{4}$ is a co-factor that regulates $\mathrm{NO}$ signalling by promoting eNOS coupling. The impact of combined treatment with $\mathrm{L}$-citrulline and $\mathrm{BH}_{4}$ on $\mathrm{NO}$ signalling is not yet clear. We tested the hypothesis that combined treatment with L-citrulline and $\mathrm{BH}_{4}$ improves eNOS coupling in hypoxic PAEC more markedly than either treatment alone.

Methods: We measured NO production, superoxide generation, and eNOS dimer-to-monomer ratios (which reflect eNOS coupling) in PAECs from newborn piglets cultured under normoxic and hypoxic conditions in the presence and absence of L-citrulline $(1 \mathrm{mM}), \mathrm{BH}_{4}(20 \mu \mathrm{M})$, or co-treated with both L-citrulline and $\mathrm{BH}_{4}$. Some studies were performed in the presence of the NOS antagonist, L-NAME. Results: NO production, superoxide generation, and eNOS dimer-to-monomer ratios improved with all treatments but the impact was greatest in hypoxic PAEC incubated with the combination of L-citrulline and $\mathrm{BH}$ than with either therapy alone. In the presence of L-NAME, the effect on superoxide generation from any therapy given singly or combined was much less than observed in the absence of L-NAME 
Conclusion: Combined treatment with L-citrulline and $\mathrm{BH}_{4}$ synergistically re-couples eNOS, more fully restoring NO signalling in hypoxic PAEC than either treatment alone. The therapeutic potential of combined therapy with L-citrulline and $\mathrm{BH}_{4}$ on the development or reversal of pulmonary hypertension in infants suffering from cardiopulmonary conditions associated with hypoxia merits future exploration.

Support: NIH Grant R56HL097566

\section{P2-8: Poor maternal nutrition during gestation alters pancreas development and function in} offspring

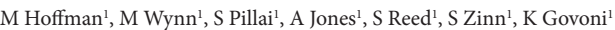

${ }^{1}$ University of Connecticut, Storrs, CT, USA

Objective: To evaluate the effects of maternal nutrition during gestation on offspring pancreas development we quantified 1) glucagon, somatostatin, and insulin positive area and cellular proliferation in offspring pancreas tissue at day (d) 90 and 135 of gestation and at birth and 2) islet number and size at $\mathrm{d} 135$.

Methods: Western Whiteface ewes were fed $100 \%$ (CON), $60 \%$ (RES) or $140 \%$ (OVER) of National Research Council requirements beginning at day $30.2 \pm 0.2$ of gestation. Offspring were necropsied at $\mathrm{d} 90$ or 135 of gestation or within 24 hours of birth. Body and pancreas weights were recorded and histological analyses via immunohistochemistry and harris hematoxylin and eosin staining were performed on the pancreas ( $n=8$ to 9 offspring per treatment per time point). ImageJ was used for quantification. Statistical analyses were performed using ANOVA.

Results: At d 135 of gestation, OVER offspring exhibited 69\% greater insulin positive area com pared with CON $(\mathrm{P} \leq 0.03)$. At $\mathrm{d} 90$ of gestation and birth no differences in insulin positive area were observed $(P \geq 0.54)$. At $d 135$ of gestation, RES and OVER offspring exhibited $38 \%$ and $63 \%$, respectively greater islet size than $\mathrm{CON}(\mathrm{P} \leq 0.01)$. Additionally, islet number was reduced $18 \%$ in OVER offspring compared with CON $(\mathrm{P}=0.03)$. In OVER offspring at birth, cell proliferation $(\mathrm{P}=0.01)$ and beta cell proliferation $(\mathrm{P}=0.01)$ were reduced compared with $\mathrm{CON}$; however, no differences were observed at $\mathrm{d} 90$ or 135 of gestation $(\mathrm{P} \geq 0.44)$. No differences were observed in offspring body weight, pancreas weight as a percent of body weight, nuclei number, and glucagon or somatostatin positive area $(\mathrm{P} \geq 0.11)$

Conclusion: Maternal under- and over-nutrition during gestation affects the structure and insulin production in the pancreas of offspring during gestation, which may contribute to altered postnatal pancreas function.

Support: USDA-AFRI-NIFA Grant 2014-01982.

P2-9: Developmental programming of pulmonary vascular dysfunction by perinatal hypoxia is sex dependent

CG Julian ${ }^{1}$, G Wolfson ${ }^{1}$, D Park ${ }^{2}$, IV Yang ${ }^{1}$, DA Schwartz

${ }^{1}$ University of Colorado, Aurora, CO, USA, ${ }^{2}$ University of Colorado Denver, Denver, CO, USA

Perinatal exposures alter susceptibility to adult-onset disease. Since developmental processes that are required for efficient pulmonary gas transfer are incomplete at birth, the lung and its pulmonary circulation are particularly vulnerable. Our human studies demonstrate that perinatal hypoxia (PHx) raises the risk of pulmonary vascular dysfunction (PVD) among male high-altitude residents.

Objective: To utilize a murine model in which the degree and timing of environmental hypoxia could be strictly controlled to directly test the hypothesis that PHx increases susceptibility to hypoxia-induced PVD during adulthood.

Methods: Experiments were performed using three groups of C57/BL6 mice distinguished by the timing of normoxic ( $\mathrm{Nx}$ ) or hypoxic $(\mathrm{Hx})$ exposure: $\mathrm{Nx}$ controls (C-C), $\mathrm{PHx}+$ adult $\mathrm{Hx}(\mathrm{PHx}-\mathrm{Hx}$ ) and perinatal $\mathrm{Nx}+$ adult $\mathrm{Hx}(\mathrm{C}-\mathrm{Hx})$. For the perinatal normoxia and $\mathrm{Hx}(\mathrm{PHx}$ or $\mathrm{Hx})$, pregnant dams were placed in a hyperbaric $(760 \mathrm{mmHg})$ or hypobaric chamber $(375 \mathrm{mmHg})$ from gestational day 14 through postnatal day 4 . All animals were housed in normoxia from postnatal day 5 to 3 weeks. Adult exposures lasted from 3-8 weeks of age. Pulmonary hemodynamic (via echocardiography) and right ventricular systolic pressure (RVSP) measurements were performed in $8 \mathrm{wk}$ old offspring. Intracardiac blood samples were used to measure hematocrit. ANOVA with multiple comparisons was used to identify differences between groups.

Results: PHx reduced pulmonary artery acceleration time and pulmonary valve peak flow velocity, and increased RV wall thickness, RVSP and hematocrit in male pups only.

Conclusion: Our studies demonstrate that PHx increases hypoxia-induced PVD, and that such effects are sex dependent.

Support: 5 K12 HD057022-07.

P2-10: Perinatal environmental stress and western diet impair postnatal lung development in mice

PY Lai ${ }^{1}, \mathrm{X}$ Jing ${ }^{1}$, T Michalkiewicz ${ }^{1}$, X Ke ${ }^{1}$, A Majnik ${ }^{1}$, RH Lane ${ }^{1}$, GG Konduri ${ }^{1}$

${ }^{1}$ Medical College of Wisconsin, Milwaukee, WI, USA

Objective: Prenatal stress and poor diet can lead to intrauterine growth restriction, which is linked to increased risk of bronchopulmonary dysplasia in preterm infants. The unique effects of environmental stress (ES) and western type diet (WD) on pre- and postnatal growth and lung development remain unknown. We hypothesize that perinatal exposure of the mother to ES and WD lead to growth restriction and impaired lung growth in the offspring in a mouse model.

Methods: Female mice were placed on either control diet (CD) or WD high in saturated fat, cholesterol, and sugar. After 5 weeks, female mice were mated and assigned to no ES or pre- and postnatal ES. Environmental stress involved maternal environmental changes like frequent cage changes and minimal bedding. Pups were weaned at postnatal day 21 (P21) and were euthanized either at P21 or P100 after receiving only CD and no ES since P21. Lungs were harvested, fixed, and stained for radial alveolar count (RAC), mean linear intercept (MLI) and capillary count (CC). Isolated RNA from lungs was analyzed by RT-PCR for genes involved in lung growth, such as VEGF and epigenetic modifications of DNA, such as DNA-methyltransferases-1\& 3a (DNMT-1, 3a)

Results: ES and WD decreased the postnatal weight only until P21. At both P21 and P100, ES alone decreased RAC ( $p<0.05$ from CD) and CC ( $p<0.05$ from CD). ES increased MLI ( $p<0.01$ from CD), indicating alveolar simplification. The changes persisted at P100 in WD+ES. Lung samples from WD+ES group had increased mRNA for VEGF, DNMT1\&3a compared to CD-no ES groups at P21.
Conclusion: Perinatal ES and WD lead to sustained inhibition of lung alveolar and vascular growth in this mouse model. This phenotype persists into adult life despite normalization of the environ ment. Perinatal stress and diet exert key influences on lung development, potentially by epigenetic changes in gene expression.

Support: RO1 HL 057268, CRI, Muma Endowed Chair of Neonatology (GGK).

P2-11: Relationships between pig weights at birth and weaning and expression of EGR1, Pref1, Cox1 and Cox2 in Wharton's jelly and the epididymal fat pad

J Morton ${ }^{1}$, T Rathbun ${ }^{1}$, DL Davis

${ }^{1}$ Kansas State University, Manhattan, KS, USA

Objective: Our goal is to determine whether low birth weight in pigs is associated with altered expression of Cox1, Cox2, Pref1 and the immediate early gene EGR1 in their Wharton's jelly and epididymal fat pads (EFP).

Methods: Umbilical cords $(n=57)$ were collected at birth and EFPs $(n=29)$ collected at castration on postnatal day 7. Birth and weaning weights (d 21) were also collected from 5 litters (PIC; $326 \times 1050)$. Wharton's jelly and EFP were snap frozen and stored $\left(-80^{\circ} \mathrm{C}\right)$ until RNA extraction (Trizol). RNA quantity (Nanodrop) and quality (Agilent Bioanalyzer) were determined. Quantitative PCR was used to determine expression of EGR1, Pref1, Cox1 and Cox2 (18S housekeeping gene).

Results: In EFP, EGR1 expression decreased with increasing birth weight $(\mathrm{P}<0.008)$ and wean weight $(\mathrm{P}<0.002)$ in agreement with our earlier observations for this gene in Wharton's jelly. Wean weight and Cox 2 expression in Wharton's jelly were negatively $(\mathrm{P}=0.05)$ correlated. No relationship between birth and wean weights for Pref1 or Cox1 $(P>0.10)$ expression were found in either tissue.

Conclusion: Wharton's jelly and EFP are sites containing mesenchymal stem cells (MSCs). Atrisk pigs have altered expression of EGR1 and Cox2 in these sites. MSCs are precursors of adipogenic cells and other mesodermal lineages and the alterations reported here may be important for programming adipose differentiation in low birth weight pigs. EGR1 expression has been shown to be altered in Wharton's jelly of small for gestational age babies. We have found that pigs weighing less than $1.11 \mathrm{~kg}$ at birth are at increased risk for pre-weaning mortality and others have shown that light birth weight pigs grow slower and are fatter at market weight. Altered expression of EGR1 and Cox2 in MSCs could indicate a role for these genes in the phenotypes reported for low birth weight pigs.

P2-12: Maternal triclosan consumption predisposes to metabolic syndrome in Wistar rat offspring

MB Rabaglino ${ }^{1}$, MJ Moreira-Espinoza ${ }^{1}$, JP Lopez ${ }^{1}$, NH Garcia ${ }^{1}$, D Beltramo

National Scientific and Technical Research Council, Córdoba, Argentina

Triclosan (TCS) is a synthetic antibacterial compound utilized in cosmetic industry and considered safe for its use in humans. The objective of this study was to evaluate the effect of maternal oral TCS intake during gestation and lactation on the metabolic status of the adult offspring. Pregnant Wistar rats were fed ad-libitum with ground food + TCS $(1 \mathrm{mg} / \mathrm{kg})$ from day $14 \mathrm{of}$ gestation to day 20 of lactation $(n=3)$ or ground food $(n=3)$. Litter size was reduced to 4 males and 4 females after born. Thus, 12 males and 12 females born from the TCS exposed rats (TCS, $\mathrm{n}=24$ ) or not (Control, $\mathrm{n}=24$ ) composed the experimental groups. Monthly body weight, food intake at 2 and 5 months and plasmatic levels of cholesterol, glucose and triglycerides at 8 months of age were measured. The effect of group on each variable was analyzed by t-test for each gender and age. Body weight was significantly heavier for males in the TCS group at 4 months $(515.6 \pm 38.3$ grs versus $451.25 \pm 51.1$ grs, $\mathrm{p}<0.001)$ and for females at 8 months $(378.3 \pm 20.2$ grs versus $306.6 \pm 20.8 \mathrm{grs}, \mathrm{p}=0.01$ ). Food intake at 5 months was significantly higher for both male and female rats in the TCS group $(52.75 \pm 3.8$ grs versus $41.25+3.3$ grs, $\mathrm{p}<0.01$ and $34.6 \pm 2.5$ grs versus $28.15 \pm 2.0$ grs, $\mathrm{p}=<0.01$ ). Cholesterol and glucose levels were significantly higher for both males and females in the TCS group (Cholesterol: $176 \pm 50.1 \mathrm{mg} / \mathrm{dl}$ versus $106.5 \pm 5.2 \mathrm{mg} /$ $\mathrm{dl}, \mathrm{p}=0.03$ and $130.5 \pm 8.1 \mathrm{mg} / \mathrm{dl}$ versus $92.2 \pm 7.3 \mathrm{mg} / \mathrm{dl}, \mathrm{p}<0.01$; Glucose: $192 \pm 55.7 \mathrm{mg} / \mathrm{dl}$ versus $117.7 \pm 17.2 \mathrm{mg} / \mathrm{dl}, \mathrm{p}=0.04$ and $175 \pm 38.8 \mathrm{mg} / \mathrm{dl}$ versus $107.7 \pm 9.46 \mathrm{mg} / \mathrm{dl}, \mathrm{p}=0.01)$. In conclusion, low doses of oral TCS consumption by the pregnant and lactating dam alter the metabolic status of the adult offspring, resembling development of the metabolic syndrome (hyperphagia, overweight, hypercholesterolemia and hyperglycemia).

P2-13: Maternal subnutrition during late gestation affects uterine development in the lamb

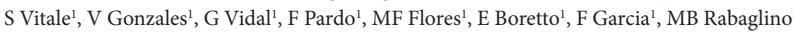
${ }^{1}$ Universidad Nacional de Rio Cuarto, Río Cuarto, Córdoba, Argentina

Nutritional restrictions during late gestation could lead to fetal hypoglycemia. Glucose levels in the fetal sheep regulate circulating insulin-like growth factor 1 (IGF-1) levels, which stimulate cell proliferation and differentiation of reproductive organs after binding its own receptor or estrogen receptors. The objectives of this study were to determine plasma levels of glucose and IGF-1 during the first 2 months of age and mRNA expressions of IGF-1 receptor (IGF1R) and estrogen receptors alpha (ESR1) and beta (ESR2) in uterus and ovaries of the neonatal lamb born from a nutritionally restricted ewe. Pregnant ewes carrying singleton females were randomly assigned to Restricted $(n=4)$ or Control $(n=4)$ groups. Ewes in the Restricted group were fed with $50 \%$ of metabolic energy requirements from $\sim 100$ days of gestation to term ( 147 days). Blood samples from lambs were taken on the first day after born and every week for plasma glucose and IGF-1 determination. Lambs were euthanatized at 2 months of age, reproductive organs were weighted and tissue samples were collected from them to measure mRNA expression by qRTPCR. Lambs born from ewes in the Restricted group showed permanent lower plasma levels of glucose and IGF-1. However, the difference was statistically significant $(\mathrm{p}<0.05)$ only on day 1 (glucose: $72.3 \pm 16.8$ versus $104.6 \pm 11.5 \mathrm{mg} / \mathrm{dl}$, IGF-1: $157.5 \pm 54.1$ versus $363 \pm 45.4 \mathrm{ng} / \mathrm{ml}$ ) and between days 7 and 10 of age for IGF-1 $(255+76.2$ versus $354.8 \pm 59.8 \mathrm{ng} / \mathrm{ml})$. These lambs also had significant lower uterine size and weight $(2.8 \pm 1.6$ versus $4.9 \pm 1.5 \mathrm{gr})$ and significant lower ovarian and uterine mRNA expression of IGF1R (down-regulation by 6.5 and 3.8 folds, respectively, compared to controls). In conclusion, maternal subnutrition during late gestation affects circulating levels of glucose and IGF-1 and impairs uterine development in the neonatal lamb, which could have permanent negative consequences in the future reproductive performance of the offspring. 
P2-14: Invasive mechanical ventilation induces necrotizing enterocolitis (NEC) and dysregulates peroxisome proliferator-activator receptor gamma (PPAR $\gamma)$ in the ileum of chronically ventilated preterm lambs

L Ruybal $^{1}$, L Dong ${ }^{1}$, M Dahl $^{1}$, K Albertine ${ }^{1}$, L Joss-Moore

${ }^{1}$ University of Utah, Salt Lake City, UT, USA

Preterm neonates supported by invasive mechanical ventilation (IMV) often have feeding intolerance and are at increased risk of developing necrotizing enterocolitis (NEC). Data are limited comparing the effect of IMV to NIS on the risk for NEC. Agonists of PPAR $\gamma$, a nutrient-responsive transcription factor, protect against NEC. A transcriptional target of PPAR $\gamma$ that may be important in NEC development is the histone methyltransferase, Setd8. The effect of mode of ventilation on PPAR $\gamma$ and Setd8 protein abundance in the ileum is unknown. We hypothesized that preterm lambs supported by IMV will develop a NEC-like phenotype after 21 days and have lower ileal PPAR $\gamma$ and Setd8 protein abundance compared to lambs supported by NIS. Preterm lambs were managed by IMV or NIS for $3 \mathrm{~d}$ or 21d (term equivalent age). Groups were fed ewe's colostrum and mature milk. Immunoblots were used to measure PPAR $\gamma$ and Setd8 protein abundance in ileal homogenate. Immunohistochemistry (IHC) was used to localize PPAR $\gamma$ in ileal sections. Gestational age (days) and terminal weight $(\mathrm{kg})$ were not different between $21 \mathrm{~d}$ groups. All of the lambs in the 21d IMV group developed NEC, including Bell Stage 3. None of the lambs in the 21d NIS group developed NEC. PPAR $\gamma$ protein abundance was not different in $21 \mathrm{~d}$ IMV compared to $21 \mathrm{~d}$ NIS ( $68 \pm 35 \%$ ). Setd8 was greater in $21 \mathrm{~d}$ IMV compared to $21 \mathrm{~d}$ NIS $(252 \pm 47 \% ; \mathrm{p}<0.05)$. PPAR $\gamma$ was lower in $3 \mathrm{~d}$ IMV compared to $3 \mathrm{~d}$ NIS $(57 \pm 17 \%$; $<0.05)$. Setd 8 was not different in $3 \mathrm{~d}$ IMV compared to $3 \mathrm{~d}$ NIS $131 \pm 24 \%)$. IHC of PPAR $\gamma$ appears less in the villi of 3d IMV lambs compared to NIS. IMV induces NEC in 21d lambs; NIS does not. Preterm lambs supported by IMV have dysregulated expression of PPAR $\gamma$ and SETD8. We speculate that increased SETD8 protein abundance may be associated with aberrant histone methylation in ileum of preterm lambs managed by IMV.

P2-15: Impact of the extracellular superoxide dismutase (EC-SOD) R213G polymorphism on alveolar and pulmonary vascular development

L Sherlock ${ }^{1}$, A Trumpie ${ }^{1}$, J Maltzahn ${ }^{1}$, C Woods ${ }^{1}$, C Wright ${ }^{1}$, C Delaney ${ }^{1}$, E Nozik Grayck ${ }^{1}$

${ }^{1}$ University of Colorado, Aurora, CO, USA

Objective: Extracellular superoxide dismutase (EC-SOD), a key vascular antioxidant enzyme, is highly localized to the extracellular matrix. An EC-SOD polymorphism leads to a single amino acid substitution (R213G), changing tissue binding affinity. This SNP increases risk for cardiovascular disease, but decreases risk for lung injury in humans and in mice with knock-in of the human R213G EC-SOD. The impact of this polymorphism on the developing lung and pulmonary circulation is unknown. We hypothesized that the R213G polymorphism will disrupt vascular development but protect alveolar development in a neonatal murine model of BPD.

Methods: Wild type (WT) mice and homozygous R213G mice were injected with intraperitoneal bleomycin $(3 \mathrm{u} / \mathrm{kg})$ or PBS three times a week for three weeks beginning on day 2 , and harvested at 22 days. Lung sections were immunostained with factor VIII or alpha smooth muscle actin. Alveolar development was evaluated by radial alveolar counts and vascular development by vessel density per high powered field. RVH, a surrogate for $\mathrm{PH}$, was determined by Fulton's index. Data were analyzed by t-test or 2-way ANOVA. Significance defined as $\mathrm{p}<0.05$.

Results: RAC was similar in WT and R213G mice at baseline. Bleomycin decreased RAC in WT mice $(\mathrm{p}<0.01)$ but not R213G mice. Vessel density was decreased in R213G mice at baseline compared to WT $(\mathrm{p}<0.05)$. Bleomycin decreased vessel density in WT mice, but did not further decrease vessel density in R213G mice ( $\mathrm{p}<0.001)$. RVH was present in R213G mice at baseline $(\mathrm{p}<0.01)$. Bleomycin treatment increased RVH in WT mice, $(\mathrm{p}<0.001)$, however did not further increase RVH in R213G mice.

Conclusion: These results indicate that a change in the distribution of EC-SOD due to the R213G SNP leads to the development of $\mathrm{PH}$ at baseline but protects mice against bleomycin-induced $\mathrm{PH}$ and abrupted alveolar development. This demonstrates that both overall EC-SOD levels and ECSOD localization are important in disease progression.

Support: T32007186-32, NIHL086680
P2-16: Maternal high fat diet during lactation permanently alters hypothalamic innervation of offspring

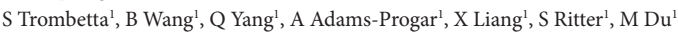

${ }^{1}$ Washington State University, Pullman, WA, USA

Maternal high fat diet (HFD) is known to induce hyperphagia in offspring, but the underlying neurological changes remain poorly defined. The innervations of pro-opiomelanocortin (POMC) and neuropeptide Y (NPY) neurons from the arcuate nucleus to other areas of the hypothalamus are an important part of the formation of the feeding circuit. These neuron innervations develop during the time period immediately after birth until the third week of life. To test the impact of maternal HFD during lactation on offspring hypothalamic development, at birth, C57BL/6 J maternal mice were fed either a control diet (10\% energy from fat) or a HFD ( $45 \%$ energy from fat) to weaning. Then, offspring female mice were fed a conventional rodent diet or a HFD to 3,6 , and 15 months of age in order to analyze long-term impact of early brain development on offspring. At 3 months of age, fat mass, fat pad weight, as well as blood glucose and triglyceride levels were significantly increased $(\mathrm{P}<0.05)$ in HFD offspring when compared with control mice. The hypothalamus was sampled for analysis at necropsy for mice aged 6 and 15 months. Mice were euthanized in the morning approximately 6 months or 15 months after birth. Brains of 6 month mice were perfused using paraformaldehyde and used for structural analysis. Brains of 15 month mice were sampled immediately after euthanization and the hypothalamus was manually extracted for PCR analysis. Compared with control mice, pro-opiomelanocortin (POMC) gene expression was reduced $(\mathrm{P}<0.05)$ in HFD offspring mice at 15 months of age. In addition, a trend of decrease in neuropeptide $\mathrm{Y}(\mathrm{NPY})$ gene expression $(\mathrm{P}<0.10)$ was also observed in HFD mice. These data indicate a negative life-long effect of HFD consumption on hypothalamic brain function in offspring.

P2-17: Vasoprotective and anti-inflammatory effects of ketamine on various brain regions of ovine fetuses subjected to hypoxic hypoxia $(\mathrm{HH})$

M Zarate ${ }^{1}$, E Chang $^{1}$, T Arndt ${ }^{1}$, C Wood ${ }^{1}$

${ }^{1}$ University of Florida, Gainesville, FL, USA

Objective: We have previously reported that hypoxic hypoxia $(\mathrm{HH})$ produces a robust inflammatory response in different brain areas, and that ketamine (NMDA receptor antagonist) reduces these effects. In order to understand the mechanism of how this inflammation occurs, we determined the distribution of albumin (blood vessel integrity marker), and IBA-1 (macrophage marker), in different brain regions of ovine fetuses exposed to $\mathrm{HH}(30 \mathrm{~min})$, with and without ketamine pretreatment.

Methods: Paraffin embedded fetal brain tissues (medulla, hypothalamus, brain cortex, and hippocampus) were sectioned ( $5 \mathrm{um}$ ) and immunostained for albumin or IBA-1, using a peroxidase-PAP sandwich technique. Broken blood vessels and macrophages were quantified in multiple randomly selected fields at 10x for albumin and 40x for IBA-1. Data were analyzed as a 2 x 2 factorial design $(\mathrm{n}=4$ per group) with a generalized linear model with Poisson distribution for log count data observations. Significance was declared at $P<0.05$ with Bonferroni post hoc correction for comparison of means.

Results: In the hypothalamus and brain cortex, $\mathrm{HH}$ produced a significant higher number of ruptured blood vessels compared to control animals, and ketamine was able to attenuate this effect ( $P=0.012$ and $P<0.001$, respectively). There was no effect of ketamine on the blood vessel integrity in the brain medulla. In the hippocampus, as we previously reported in hypothalamus and cerebral cortex, $\mathrm{HH}$ produced significantly higher density of IBA-1 positive cells in CA1, CA2, and CA3 $(P<0.01, \mathrm{P}<0.001$, and $\mathrm{P}<0.01$, respectively) regions compared to control; this response was reduced by the administration of ketamine.

Conclusion: We conclude that HH ruptures - and ketamine protects -- blood vessels in the fetal cerebral cortex and hypothalamus, with influx of macrophages or redistribution of microglia. Hypoxic hypoxia compromises the integrity of the blood brain barrier and produces an inflammatory response, and ketamine reduces both the damage and inflammation.

Support: NIH Grant HD033053 